\title{
Distances dans la suite des multiples d'un point du tore à deux dimensions
}

\author{
par
}

\author{
Nicolas Chevallier (Mulhouse)
}

Introduction. Soit $\theta$ un élément de $\mathbb{T}^{1}=\mathbb{R} / \mathbb{Z}$. Considérons la suite des multiples de $\theta, x=(n \theta)_{n \in \mathbb{N}}$. Pour tout $n \in \mathbb{N}$, ordonnons les $n+1$ premiers termes de cette suite, $\left\{0=y_{0} \leq y_{1} \leq \ldots \leq y_{n} \leq 1\right\}=\{p \theta, p=0, \ldots, n\}$. La suite $\left(y_{0}, \ldots, y_{n}\right)$ découpe l'intervalle $[0,1]$ en $n+1$ intervalles qui ont au plus trois longueurs distinctes, la plus grande de ces longueurs étant la somme des deux autres. Cette propriété a été conjecturé par Steinhaus, elle est étroitement liée au développement en fraction continue de $\theta$. On peut aussi la démontrer directement [So]. Lorsque $n=q_{k}-1$, où $q_{k}$ est le dénominateur d'une réduite de $\theta$, les intervalles n'ont que deux longueurs possibles, la plus grande étant inférieure au double de la plus petite. Ainsi, la distance du milieu d'un grand intervalle, à la suite $(p \theta)_{p \leq n}$, est inférieure à la longueur d'un petit intervalle. Si $\theta$ est irrationnel, la suite $(n \theta)_{n \in \mathbb{N}}$ vérifie donc la propriété suivante (avec $c=1)$ :

Il existe une infinité d'entiers $n$ tels que la distance maximale des points du tore à la suite $(p \theta)_{p \leq n}$ soit comparable à la distance mutuelle des points les plus rapprochés de la suite $(p \theta)_{p \leq n}$, i.e., $\exists c \in \mathbb{R} \exists I$ infini $\subseteq \mathbb{N}, \forall n \in I$,

$\sup \left\{d(x,\{0, \theta, \ldots, n \theta\}): x \in \mathbb{T}^{1}\right\} \leq c \inf \{d(p \theta, q \theta): p \neq q$ et $0 \leq p, q \leq n\}$.

Nous nous proposons dans ce travail d'étudier la même propriété pour les $\theta$ appartenant à $\mathbb{T}^{2}=(\mathbb{R} / \mathbb{Z})^{2}$. Contrairement à la dimension 1 , nous montrons qu'il existe des $\theta \in \mathbb{T}^{2}$ dont l'ensemble des multiples est dense et tels que la propriété précédente soit fausse. Nous montrons aussi que l'ensemble de ces $\theta$ est de mesure nulle pour la mesure de Lebesgue de $\mathbb{T}^{2}$. Ceci est l'objet des paragraphes 3 et 4 . Pour démontrer ce résultat, on utilise la notion de meilleure approximation de $\theta$, qui remplace le développement en fraction continue. Les meilleures approximations nous permettent, en modifiant légèrement $\theta$, de nous ramener à l'étude de sous-groupes finis de $\mathbb{T}^{2}$. Dans les paragraphes 1 et 2 on donne deux lemmes, l'un sur les meilleures approximations d'un couple d'irrationnels, l'autre sur la répartition des sous- 
groupes finis de $\mathbb{T}^{2}$. On en déduit des corollaires simples sur le nombre de distances minimales (corollaire 1.2(2)) dans la suite $(p \theta)_{p \leq n}$ et sur la répartition de cette suite lorsque $\theta$ est mal approximable.

\section{Notations et définitions}

1. Si $x$ est un réel, $\|x\|,[x]$ et $\{x\}$ désignent respectivement la distance à l'entier le plus proche, le plus grand entier inférieur à $x$ et la partie fractionnaire de $x$, i.e., $\{x\}=x-[x]$. Si $x$ est un élément de $\mathbb{T}^{1},\|x\|$ désigne la distance de $x$ à 0 dans $\mathbb{T}^{1}$.

2. Pour $x=(y, z) \in \mathbb{T}^{2}$, on note

$$
\begin{gathered}
\|x\|=\sup (\|y\|,\|z\|), \quad r(n, x)=\inf \{\|k x\|: k=1, \ldots, n\}, \\
e(n, x)=\sup \left\{d(u,\{0, x, 2 x, \ldots, n x\}): u \in \mathbb{T}^{2}\right\} .
\end{gathered}
$$

Remarques. (a) Pour $k \in \mathbb{N}$ et $x \in \mathbb{T}^{2}$, on a $\|k x\| \leq k\|x\|$.

(b) On notera de la même manière un élément de $\mathbb{R}^{2}$ et sa classe dans $\mathbb{T}^{2}$. Si $x=(a, b)$ est un élément de $\mathbb{R}^{2}$ tel que $\sup (|a|,|b|)=\|x\|$ alors pour tout $\lambda \in \mathbb{R}^{+}$on a $\|\lambda x\| \leq \sup (|\lambda a|,|\lambda b|)=\lambda\|x\|$.

3. Soit $\theta \in \mathbb{T}^{2}$. Appelons $q_{n}(\theta)$ le $n$ ième entier $p$ tel que $\|p \theta\|<\|k \theta\|$ pour tous les $k$ compris entre 1 et $p-1\left(q_{1}(\theta)=1\right)$. Lorsqu'il n'y a pas d'ambiguïté, on note simplement $q_{n}(\theta)$ par $q_{n}$. Posons aussi $r_{n}=r_{n}(\theta)=\left\|q_{n} \theta\right\|$. Nous dirons que $q_{n}$ est le dénominateur d'une meilleure approximation, ou plus simplement, une meilleure approximation.

4. Si $H$ est un sous-groupe d'un groupe $H^{\prime}$ muni d'une distance $\delta$, on note

$$
r(H)=\inf \{\delta(x, 0): x \in H \backslash\{0\}\} \quad \text { et } \quad e(H)=\sup \left\{\delta(x, H): x \in H^{\prime}\right\} .
$$

5. Si $x$ est un élément d'un groupe $H$, on note $\langle x\rangle$ le sous groupe engendré par $x$.

6. Si $H$ est un groupe muni d'une distance, $B^{*}(x, r)$ désigne la boule de centre $x$ et de rayon $r$, privée de $x$.

7. Si $n$ est un entier $d(n), \phi(n)$ et $\sigma(n)$ désignent les fonctions arithmétiques usuelles, c'est-à-dire,

$$
d(n)=\sum_{d \mid n} 1, \quad \sigma(n)=\sum_{d \mid n} d, \quad \phi(n)=\sum_{d \wedge n=1} 1 .
$$

1. Remarques sur les meilleures approximations. Les meilleures approximations d'un élément du tore ont déja été étudiées par de nombreux auteurs. Le (2) du lemme suivant n'est qu'un affaiblissement d'un résultat de J. C. Lagarias ([L]); nous en donnons la démonstration qui est très simple. Ce lemme établit un lien entre les meilleures approximations en dimension 
2 et le développement en fraction continue en dimension 1. Le (1) est une remarque très utile dans la suite.

Lemme 1.1. Soit $\theta \in \mathbb{T}^{2}$. On a

(1) $q_{n+1} r_{n}^{2} \leq 1$,

(2) $q_{n+4}>2 q_{n}$

(3) $\left[q_{n+1} / q_{n}\right] \geq\left(r_{n-1}-r_{n+1}\right) / r_{n}$ pour $n \geq 3$.

Démonstration. (1) Il suffit de remarquer que dans $\mathbb{T}^{2}$, les boules ouvertes $B\left(k \theta, r_{n} / 2\right), k=0, \ldots, q_{n+1}-1$, sont disjointes.

(2) Si $q_{n+4} \leq 2 q_{n}$ alors pour $i, j=0,1, \ldots, 4$ on a $q_{n+i}-q_{n+j} \leq q_{n}$. Donc $\left\|q_{n+j} \theta-q_{n+i} \theta\right\| \geq r_{n}$, ce qui est impossible car la boule $B\left(0, r_{n}\right)$ ne peut pas contenir 5 points dont les distances mutuelles sont supérieures à $r_{n}$ et dont 4 sont dans l'intérieur.

(3) On a $r_{n+1}=\left\|q_{n+1} \theta\right\|=\left\|\left[q_{n+1} / q_{n}\right] q_{n} \theta+q \theta\right\| \geq r_{n-1}-\left[q_{n+1} / q_{n}\right] r_{n}$ si $q>0$. Si $q=0$ alors $q_{n}$ divise $q_{n+1}$. Comme $r_{n}<r_{n-1}<1 / 2$, en raisonnant sur les composantes de $\theta$, on montre que $q_{n+1} / q_{n} \geq\left[1 / r_{n}\right] \geq 1 /\left(2 r_{n}\right) \geq$ $r_{n-1} / r_{n}$.

Corollaire 1.2. (1) Soit $\theta \in \mathbb{T}^{2}$ un couple mal approximable, c'est-àdire, tel que $\inf \left\{n\|n \theta\|^{2}: n \in \mathbb{N}^{*}\right\}=c>0$. On a $q_{n+1} / q_{n} \leq 1 / c$ pour tout $n \in \mathbb{N}^{*}$.

(2) Soient $\theta \in \mathbb{T}^{2}$ et $N$ un entier. Pour $q=0, \ldots, N$ notons $D_{q}=$ $d(q \theta,\{0, \theta, \ldots, N \theta\} \backslash\{q \theta\})$. On a $\operatorname{card}\left(\left\{D_{q}: q=0, \ldots, N\right\}\right) \leq 5$.

Démonstration. (1) D'après le lemme 1.1(1), on a $q_{n} r_{n}^{2} / c \geq 1 \geq$ $q_{n+1} r_{n}^{2}$.

(2) C'est une conséquence du lemme 1.1(2) et du résultat suivant.

Lemme 1.3. Soient $(X, d)$ un espace métrique, $f$ une isométrie de $X$ dans $X$ et $x_{0}$ un point de $X$. Posons $x_{q}=f^{q}\left(x_{0}\right), q_{1}=1, q_{n+1}=\inf \{q>$ $\left.q_{n}: d\left(x_{0}, x_{q}\right)<d\left(x_{0}, x_{q_{n}}\right)\right\}$. Soit $N$ un entier. Notons $D_{q}=d\left(x_{q},\left\{x_{0}, \ldots\right.\right.$ $\left.\left.\ldots, x_{N}\right\} \backslash\left\{x_{q}\right\}\right)$. Si $n$ et $m$ sont deux entiers tels que $q_{n} \leq N<q_{n+1}$ et $2 q_{m} \leq N<2 q_{m+1}$ alors

$$
\operatorname{card}\left(\left\{D_{q}: q=0, \ldots, N\right\}\right)= \begin{cases}n-m & \text { si } 2 q_{m+1}=N+1 \\ n-m+1 & \text { si } 2 q_{m+1}>N+1 .\end{cases}
$$

Démonstration du lemme. Notons $d_{i}=d\left(x_{0}, x_{q_{i}}\right)$. Pour chaque $q$, appelons $h(q)$ un indice tel que $D_{q}=d\left(x_{q}, x_{h(q)}\right)$. En changeant éventuellement $h(q)$ on peut supposer que $|q-h(q)|=q_{i}$ pour un certain $i \in$ $\{1, \ldots, n\}$. On en déduit

(i) si $q \leq N-q_{i}$ ou $q \geq q_{i}$ alors $D_{q} \leq d_{i}$,

(ii) si $N-q_{i}<q<q_{i}$ alors $D_{q}>d_{i}$. 
On a $0 \leq N-q_{n}<N-q_{n-1}<\ldots<N-q_{m+1}<q_{m+1}<\ldots<q_{n} \leq$ $N<q_{n+1}$. D'après (i) et (ii), on a

- Si $q \in\left[0, N-q_{n}\right] \cup\left[q_{n}, N\right]$ alors $D_{q}=d_{n}$.

- Si $\left.q \in] N-q_{p}, N-q_{p-1}\right] \cup\left[q_{p-1}, q_{p}[\right.$ pour $p \in\{m+2, \ldots, n\}$ alors $D_{q}=d_{p-1}$.

- Si $q \in] N-q_{m+1}, q_{m+1}\left[\right.$ alors $D_{q}>d_{m+1}$; de plus, comme $q_{m} \leq$ $N-q_{m}$, on a soit $q \leq N-q_{m}$, soit $q \geq q_{m}$, d'où $D_{q}=d_{m}$.

Re m ar que. Pour la suite $n(9 / 50,11 / 38), n=0, \ldots, 11$, dans $\mathbb{T}^{2}$, on a 4 distances minimales.

2. Répartition d'un sous groupe fini de $G=(\mathbb{R} / n \mathbb{Z}) \times(\mathbb{R} / m \mathbb{Z})$. Si $H$ est un sous groupe fini de $\mathbb{T}^{1}$, on a clairement $e(H)=r(H) / 2$. La proposition suivante donne le résultat correspondant pour les sous-groupes finis de $\mathbb{T}^{2}$. Munissons $\mathbb{R}$ de la distance usuelle et $\mathbb{R} / n \mathbb{Z}$ et $\mathbb{R} / m \mathbb{Z}$ des distances quotients. Appelons $d$ la distance sur $G$ définie par le maximum des distances sur chaque composante.

Proposition 2.1. Soit $H$ un sous groupe de $G=(\mathbb{R} / n \mathbb{Z}) \times(\mathbb{R} / m \mathbb{Z})$. Si $r(H) \leq \inf (m, n)$ on a

$$
n m /(4 r(H) \operatorname{card}(H)) \leq e(H) \leq n m /(r(H) \operatorname{card}(H)) .
$$

Démonstration. Soit $x=(r, s)$ un élément de $H$ tel que $d(x, 0)=$ $r(H)$. On peut supposer $r \geq s \geq 0$ et $r>0$. Appelons $p$ la projection de $\mathbb{R} \times \mathbb{R}$ sur $(\mathbb{R} / n \mathbb{Z}) \times(\mathbb{R} / m \mathbb{Z})$, et appelons $K$ l'image réciproque de $H$ par $p$. Alors $K$ est inclus dans $(1 / \operatorname{card}(H))(n \mathbb{Z} \times m \mathbb{Z})$. Soit $K^{\prime}=(K+\mathbb{R} x) \cap(\{0\} \times \mathbb{R})$; c'est un sous-groupe de $\{0\} \times \mathbb{R}$. On vérifie que $K^{\prime}$ est discret, on a donc $K^{\prime}=\{0\} \times(a \mathbb{Z})$ où $a$ est un réel strictement positif.

1. Soit $y=(0, a)$. Alors $x$ et $y$ forment une base de $\mathbb{R}^{2}$. Montrons que

$$
C=\left\{t x+t^{\prime} y \in \mathbb{R}^{2}: t, t^{\prime} \in[0,1[\}\right.
$$

est un domaine fondamental pour $K$.

Si $P \in \mathbb{R}^{2}$, il existe des réels $u$ et $v$ tels que $P=u x+v y$. Comme $x$ et $y$ sont des éléments de $K+\mathbb{R} x$, le point $P^{\prime}=u x+[v] y$ appartient à $K+\mathbb{R} x$, donc $P^{\prime}=g+\lambda x$ avec $g \in K$ et $\lambda \in \mathbb{R}$. D'où $P=g+[\lambda] x+\{\lambda\} x+\{v\} y \in$ $C+g+[\lambda] x$.

Soient $P=u x+v y$ et $P^{\prime}=u^{\prime} x+v^{\prime} y$ deux éléments de $C$ tels que $P-P^{\prime} \in K$. Comme $P-P^{\prime}=\left(u-u^{\prime}\right) x+\left(v-v^{\prime}\right) y$, on a $\left(v-v^{\prime}\right) y \in K+\mathbb{R} x$. De plus, $\left|v-v^{\prime}\right|<1$, la minimalité de $y$ montre donc que $v=v^{\prime}$. De même, $\left|u-u^{\prime}\right|<1$, la minimalité de $x$ montre que $u=u^{\prime}$, d'où $P=P^{\prime}$.

2. Montrons que $a=n m /(\operatorname{card}(H) r(H))$. L'ensemble des $C+y$ quand $y$ parcourt $K$ forme une partition de $\mathbb{R}^{2}$. Comme $K$ contient $(n \mathbb{Z}) \times(m \mathbb{Z})$, les $p(C)+z$, quand $z$ parcourt $H$, forment une partition de $(\mathbb{R} / n \mathbb{Z}) \times(\mathbb{R} / m \mathbb{Z})$. 
La mesure de Lebesgue sur $(\mathbb{R} / n \mathbb{Z}) \times(\mathbb{R} / m \mathbb{Z})$ est invariante par translation, donc

$$
\operatorname{aire}((\mathbb{R} / n \mathbb{Z}) \times(\mathbb{R} / m \mathbb{Z}))=\operatorname{card}(H) \times \operatorname{aire}(p(C)),
$$

et comme aire $(p(C))=\operatorname{aire}(C)=a r$, on obtient $a=n m /(\operatorname{card}(H) r(H))$.

3 . Les boules de centre un point de $H$ et de rayon $r(H) / 2=r / 2$ sont disjointes et comme $r(H) \leq m, n$, leurs aires valent $r(H)^{2}$, donc $r(H) \leq$ $n m /(r(H) \operatorname{card}(H))$.

4. Soit $M$ un point de $\mathbb{R}^{2}$ (muni de la distance sup des coordonnées). Comme $s / r \leq 1$, on a $d(M, K) \leq(a+r) / 2$ et $d((0, a / 2), K+\mathbb{R} x) \geq a / 4$. Finalement, on obtient

$$
\begin{aligned}
n m /(4 \operatorname{card}(H) r(H)) & =a / 4 \leq d((0, a / 2), K+\mathbb{R} x) \leq e(H) \\
& \leq(r+a) / 2 \leq n m /(\operatorname{card}(H) r(H)) .
\end{aligned}
$$

Application aux couples d'irrationnels

Proposition 2.2. Soit $\theta \in \mathbb{T}^{2}$. On a $e\left(q_{n}, \theta\right) \leq 3 /\left(r_{n-1}(\theta) q_{n}(\theta)\right)$.

Démonstration. Soit $x$ le représentant dans $\mathbb{R}^{2}$ de $q_{n} \theta$ le plus proche de 0 , et posons $\theta^{\prime}=\theta-x / q_{n}$. Alors $H=\left\{0, \theta^{\prime}, 2 \theta^{\prime}, \ldots,\left(q_{n}-1\right) \theta^{\prime}\right\}$ est un sousgroupe cyclique à $q_{n}$ éléments de $\mathbb{T}^{2}$. Minorons $r(H)$; soit $k \in\left\{1, \ldots, q_{n}-1\right\}$ tel que $\left\|k \theta^{\prime}\right\|$ soit minimum. Comme $k \theta^{\prime}=-\left(q_{n}-k\right) \theta^{\prime}$ on peut supposer que $k \leq q_{n} / 2$, donc

$$
\begin{aligned}
r(H) & =\left\|k\left(\theta-x / q_{n}\right)\right\| \geq\|k \theta\|-\left\|k x / q_{n}\right\| \\
& \geq r_{n-1}(\theta)-\left(k / q_{n}\right) r_{n}(\theta) \geq(1 / 2) r_{n-1}(\theta) .
\end{aligned}
$$

Grâce à la proposition 2.1, on a

$$
e\left(q_{n}, \theta^{\prime}\right)=e(H) \leq 1 /\left(q_{n} r(H)\right) \leq 2 /\left(q_{n} r_{n-1}(\theta)\right) .
$$

De plus, pour $k \in\left\{0, \ldots, q_{n}\right\}$ et $y \in \mathbb{T}^{2}$, on a

$$
\|y-k \theta\| \leq\left\|y-k \theta^{\prime}\right\|+\left\|k\left(\theta-\theta^{\prime}\right)\right\| \leq\left\|y-k \theta^{\prime}\right\|+\left(k / q_{n}\right) r_{n}(\theta),
$$

donc $e\left(q_{n}, \theta\right) \leq e\left(q_{n}, \theta^{\prime}\right)+r_{n}(\theta)$. On conclut en utilisant l'inégalité $r_{n} \leq$ $r_{n-1} \leq 1 /\left(q_{n} r_{n-1}\right)$.

Corollaire 2.3. Soit $\theta \in \mathbb{T}^{2}$ tel que $\inf \left\{n\|n \theta\|^{2}: n \in \mathbb{N}^{*}\right\}=c>0$. On a

(1) $\sup \{\sqrt{n} e(n, \theta): n \in \mathbb{N}\} \leq 3 / c$,

(2) $\sup \{e(n, \theta) / r(n, \theta): n \in \mathbb{N}\} \leq 3 / c$.

Démonstration. Soit $q_{n} \leq m<q_{n+1}$. D'après la proposition précédente, on a

$$
e(m, \theta) \leq e\left(q_{n}, \theta\right) \leq 3 /\left(r_{n-1} q_{n}\right) \leq 3 c^{-1 / 2} q_{n-1}^{1 / 2} / q_{n} .
$$

D'après le corollaire $1.2(1)$, on a $q_{n+1} / q_{n} \leq 1 / c$, d'où 


$$
\begin{gathered}
\sqrt{m} e(m, \theta) \leq q_{n+1}^{1 / 2}\left(3 c^{-1 / 2} q_{n-1}^{1 / 2} / q_{n}\right) \leq 3 / c \\
e(m, \theta) / r(m, \theta) \leq e(m, \theta) / r_{n} \leq e(m, \theta) c^{-1 / 2} q_{n}^{1 / 2} \leq 3 / c .
\end{gathered}
$$

3. Exemple d'un $\theta \in \mathbb{T}^{2}$ tel que $\lim _{n \rightarrow \infty} \sqrt{n} e(n, \theta)=\infty$ et $\theta \mathbb{N}$ soit dense dans $\mathbb{T}^{2}$. Soient $(n(k))$ et $(m(k))$ deux suites d'entiers vérifiant $n(0)=m(0)=1$ et

- si $k$ est pair, $m(k+1)=m(k)$ et $n(k+1)$ est tel que $2^{n(k+1)} \geq$ $k^{2} 2^{2 n(k)+2} 3^{3 m(k)}$,

- si $k$ est impair, $n(k+1)=n(k)$ et $m(k+1)$ est tel que $3^{m(k+1)} \geq$ $k^{2} 3^{2 m(k)+2} 2^{3 n(k)}$.

Définissons une suite $\theta(k)$ de $\mathbb{T}^{2}$ par

$$
\begin{gathered}
\theta(0)=\left(2^{-n(0)}, 3^{-m(0)}\right), \\
\theta(k+1)= \begin{cases}\theta(k)+\left(2^{-n(k+1)}, 0\right) & \text { si } k \text { est pair, } \\
\theta(k)+\left(0,3^{-m(k+1)}\right) & \text { si } k \text { est impair. }\end{cases}
\end{gathered}
$$

Notons $N(k)=2^{n(k)} 3^{m(k)}$ et

$$
\varepsilon(k)= \begin{cases}\left(2^{-n(k+1)}, 0\right) & \text { si } k \text { est pair, } \\ \left(0,3^{-m(k+1)}\right) & \text { si } k \text { est impair. }\end{cases}
$$

LeMme 3.1. $\sum_{p>k}\|\varepsilon(p)\| \leq N(k)^{-2}$.

Démonstration. Le choix des suites $n(k)$ et $m(k)$ montre que $\|\varepsilon(p)\|$ $\leq 1 /\left(4 N(p)^{2}\right)$. De plus, $N(p+1) \geq N(p)^{2} \geq 2 N(p)$, d'où

$$
\sum_{p>k}\|\varepsilon(p)\| \leq N(k)^{-2} \sum_{p>0} 2^{-p}=N(k)^{-2} .
$$

Par récurrence, on montre que $\theta(k)=\left(a(k) 2^{-n(k)}, b(k) 3^{-m(k)}\right)$, où $a(k)$ est un entier non divisible par 2 et $b(k)$ est un entier non divisible par 3 . Le sous-groupe de $\mathbb{T}^{2}$ engendré par $\theta(k)$ est donc l'ensemble des couples de la forme $\left(a 2^{-n(k)}, b 3^{-m(k)}\right)$ avec $a$ et $b$ entier, d'où

$$
2 e(N(k), \theta(k))= \begin{cases}2^{-n(k)} & \text { si } k \text { est pair, } \\ 3^{-m(k)} & \text { si } k \text { est impair. }\end{cases}
$$

Supposons $k$ pair. Soit $N^{\prime}(k)=\left[2^{n(k+1)-2 n(k)-2} 3^{-m(k)}\right]$. Si $n \in\{N(k)$, $\left.\ldots, N^{\prime}(k)\right\}$ on a

$$
\|n \theta(k+1)-n \theta(k)\| \leq N^{\prime}(k) 2^{-n(k+1)}=2^{-n(k)} / 4,
$$

d'où

$$
\begin{aligned}
e(n, \theta(k+1)) & \geq 2^{-n(k)-2} \geq(1 / \sqrt{n}) N(k)^{1 / 2} 2^{-n(k)-2} \\
& =(1 / \sqrt{n}) 2^{-(1 / 2) n(k)-2} 3^{(1 / 2) m(k)} .
\end{aligned}
$$


De plus, on a $n(k)=n(k-1)$ et $3^{m(k)} \geq(k-1)^{2} 2^{3 n(k-1)} 3^{2 m(k-1)+2}$, d'où

$$
e(n, \theta(k+1)) \geq(1 / \sqrt{n}) 2^{n(k)} .
$$

Si $n \in\left\{N^{\prime}(k), \ldots, N(k+1)\right\}$, on a

$$
\begin{aligned}
e(n, \theta(k+1)) & \geq \frac{1}{2} 3^{-m(k)} \geq \frac{1}{2}(1 / \sqrt{n}) N^{\prime}(k)^{1 / 2} 3^{-m(k)} \\
& \geq \frac{1}{2}(1 / \sqrt{n}) 2^{(1 / 2) n(k+1)-n(k)-1} 3^{(-3 / 2) m(k)} \geq(1 / \sqrt{n}) k .
\end{aligned}
$$

Lorsque $k$ est impair, un raisonnement identique montre que pour $n \in$ $\{N(k), \ldots, N(k+1)\}$, on a $e(n, \theta(k+1)) \geq(1 / \sqrt{n}) k$. Finalement, grâce au lemme 3.1 , on obtient pour $n \in\{N(k), \ldots, N(k+1)\}$

$$
\begin{aligned}
e(n, \theta) & \geq e(n, \theta(k+1))-N(k+1) N(k+1)^{-2} \\
& \geq(1 / \sqrt{n}) k-1 / n \geq(1 / \sqrt{n})(k-1) .
\end{aligned}
$$

4. Mesure de l'ensemble $\left\{\theta \in \mathbb{T}^{2}: \liminf _{n \rightarrow \infty} e(n, \theta) / r(n, \theta)<\infty\right\}$. Soient

$$
\begin{aligned}
& \mathcal{A}=\left\{\theta \in \mathbb{T}^{2}: \liminf _{n \rightarrow \infty} e(n, \theta) / r(n, \theta)<\infty\right\}, \\
& \mathcal{B}=\left\{\theta \in \mathbb{T}^{2}: \liminf _{n \rightarrow \infty} e(n, \theta) \sqrt{n}<\infty\right\}, \\
& \mathcal{C}=\left\{\theta \in \mathbb{T}^{2}: \liminf _{n \rightarrow \infty} e\left(q_{n}, \theta\right) / r_{n-1}<\infty\right\} .
\end{aligned}
$$

ThÉORÈme 4.1. Les trois ensembles $\mathcal{A}, \mathcal{B}$ et $\mathcal{C}$ sont égaux et de mesure 1 pour la mesure de Lebesgue de $\mathbb{T}^{2}$.

Plan de la démonstration. Remarquons d'abord que $\mathcal{C} \subseteq \mathcal{A} \subseteq$ $\mathcal{B}$. En effet, comme

$$
e\left(q_{n}-1, \theta\right) \leq e\left(q_{n}, \theta\right)+r_{n}(\theta) \text { et } r\left(q_{n}-1, \theta\right)=r_{n-1}(\theta),
$$

on a $e\left(q_{n}-1, \theta\right) / r\left(q_{n}-1, \theta\right) \leq e\left(q_{n}, \theta\right) / r_{n-1}(\theta)+1$, donc $\mathcal{C} \subseteq \mathcal{A}$. De plus, pour $q_{n} \leq m<q_{n+1}$, on a $\sqrt{m} r(m, \theta)=\sqrt{m} r_{n} \leq \sqrt{q_{n+1}} r_{n} \leq 1$, donc $\sqrt{m} e(m, \theta) \leq e(m, \theta) / r(m, \theta)$ et $\mathcal{A} \subseteq \mathcal{B}$.

Et ape 1 : on montre que $\mathcal{B} \subseteq \mathcal{C}$. Notons que cela utilise la minoration de la proposition 2.1 .

Et ape 2 : on montre que $\mathcal{B}$ est de mesure strictement positive. L'estimation du lemme 4.3 est de nature arithmétique, elle permet d'utiliser la majoration de la proposition 2.1. Le reste de cette étape est classique et il est inspiré par le premier chapitre du livre de Sprindžuk ([Sp]).

Etape 3 : on montre que $\mathcal{B}$ est de mesure 1 en utilisant l'ergodicité de la transformation de $\mathbb{T}^{2}, x$ donne $2 x$.

Etape 1

Lemme 4.1. Soient $\theta \in(\mathbb{R} / \mathbb{Z})^{2}$ et $m \in \mathbb{Z}$ tel que $e(m, \theta) \leq c / \sqrt{m}$. Si $q_{n}<m \leq q_{n+1}$ alors 
(1) $q_{n+1} \leq C m$ ou $m \leq C q_{n}$,

(2) $e\left(q_{n+1}, \theta\right) \leq C^{\prime} / \sqrt{q_{n+1}}$ ou $e\left(q_{n}, \theta\right) \leq C^{\prime} / \sqrt{q_{n}}$,

où $C$ et $C^{\prime}$ ne dépendent que de $c$.

Démonstration. (1) La distance sur $\mathbb{T}^{2}$ étant invariante par translation, on a

$$
d(0,\{m \theta, \ldots, 2 m \theta\}) \leq d(-m \theta,\{0, \ldots, m \theta\}) \leq e(m, \theta),
$$

donc il existe $m^{\prime} \in\{m, \ldots, 2 m\}$ tel que $\left\|m^{\prime} \theta\right\| \leq e(m, \theta)$. Choisissons un tel $m^{\prime}$, et soit $\varepsilon$ un représentant dans $\mathbb{R}^{2}$ de $m^{\prime} \theta$ dont la distance à 0 est $\left\|m^{\prime} \theta\right\|$. Posons $\theta^{\prime}=\theta-\varepsilon / m^{\prime}$. D'après la proposition 2.1 , on a

$$
e\left(m^{\prime}, \theta^{\prime}\right) \geq 1 /\left(4 m^{\prime} r\left(m^{\prime}-1, \theta^{\prime}\right)\right) .
$$

On a aussi

$$
\begin{aligned}
r\left(m^{\prime}-1, \theta^{\prime}\right) & \leq\left\|q_{n} \theta^{\prime}\right\| \leq\left\|q_{n} \theta\right\|+\left\|q_{n}\left(\theta-\theta^{\prime}\right)\right\| \leq r\left(q_{n}, \theta\right)+\left\|q_{n} \varepsilon / m^{\prime}\right\| \\
& \leq r_{n}+\left(q_{n} / m^{\prime}\right)\|\varepsilon\| \leq r_{n}+\left(q_{n} / m^{\prime}\right) e(m, \theta) \leq r_{n}+c q_{n} / m^{\prime} \sqrt{m} .
\end{aligned}
$$

D'où

$$
\begin{gathered}
2 c / \sqrt{m} \geq e(m, \theta)+\left\|m^{\prime} \theta\right\| \geq e\left(m^{\prime}, \theta^{\prime}\right) \geq 1 /\left(4 m^{\prime}\left(r_{n}+c q_{n} / m^{3 / 2}\right)\right), \\
16 c \sqrt{m}\left(r_{n}+c q_{n} / m^{3 / 2}\right) \geq 1 \\
r_{n} \sqrt{q_{n+1}} 16 c\left(m / q_{n+1}\right)^{1 / 2}+16 c^{2} q_{n} / m \geq 1 .
\end{gathered}
$$

L'un des 2 termes du premier membre de l'inégalité précédente, est donc plus grand que $1 / 2$. On conclut en remarquant que $r_{n} \sqrt{q_{n+1}} \leq 1$.

(2) Si $m \leq q_{n+1} \leq C m$, alors

$$
e\left(q_{n+1}, \theta\right) \leq e(m, \theta) \leq c / \sqrt{m} \leq c \sqrt{C} / \sqrt{q_{n+1}} .
$$

Si $q_{n} \leq m \leq C q_{n}$, alors

$$
e\left(q_{n}, \theta\right) \leq e(m, \theta)+C r_{n} \leq C(c+1) / \sqrt{q_{n}} .
$$

Lemme 4.2. (1) Soient $\theta \in \mathbb{T}^{2}$ et $n \in \mathbb{N}$ tels que $e\left(q_{n}, \theta\right) \leq c / \sqrt{q_{n}}$. On a $e\left(q_{n}, \theta\right) / r_{n-1}(\theta) \leq C$, où $C$ ne dépend que de $c$.

(2) $\mathcal{B} \subseteq \mathcal{C}$.

Démonstration. (1) Soit $\varepsilon$ un représentant dans $\mathbb{R}^{2}$ de $q_{n} \theta$ dont la distance à 0 est $\left\|q_{n} \theta\right\|$ et soit $\theta^{\prime}=\theta-\varepsilon / q_{n}$. On a

$$
r\left(q_{n}-1, \theta^{\prime}\right) \leq\left\|q_{n-1} \theta^{\prime}\right\| \leq\left\|q_{n-1} \theta\right\|+\left\|q_{n-1}\left(\theta-\theta^{\prime}\right)\right\| \leq 2 r_{n-1}(\theta) .
$$

D'après la proposition 2.1 , on a

$$
e\left(q_{n}, \theta^{\prime}\right) \geq 1 /\left(4 q_{n} r\left(q_{n}-1, \theta^{\prime}\right)\right) \geq 1 /\left(8 q_{n} r_{n-1}(\theta)\right) .
$$

De plus, $e\left(q_{n}, \theta^{\prime}\right) \leq e\left(q_{n}, \theta\right)+r_{n}(\theta) \leq 2 e\left(q_{n}, \theta\right)$, donc

$$
2 c / \sqrt{q_{n}} \geq 2 e\left(q_{n}, \theta\right) \geq 1 /\left(8 q_{n} r_{n-1}(\theta)\right) .
$$


On obtient ainsi $1 / r_{n-1} \leq 16 c \sqrt{q_{n}}$ et $e\left(q_{n}, \theta\right) / r_{n-1} \leq e\left(q_{n}, \theta\right) 16 c \sqrt{q_{n}}$ $\leq 16 c^{2}$.

(2) Soit $\theta \in \mathbb{T}^{2}$ tel que $\liminf _{n \rightarrow \infty} e(n, \theta) \sqrt{n}<c<\infty$. Il existe une infinité de $n$ tels que $e(n, \theta) \sqrt{n}<c$. D'après le lemme 4.1, il existe alors une infinité de $n$ tels que $e\left(q_{n}, \theta\right)<a / \sqrt{q_{n}}$, où $a$ ne dépend que de $c$. On conclut grâce à (1).

Etape 2. Le lemme suivant donne une minoration du nombre d'éléments de $G=(\mathbb{Z} / n \mathbb{Z}) \times(\mathbb{Z} / n \mathbb{Z})$ d'ordre $n$ qui engendrent un sous-groupe "bien réparti".

Lemme 4.3. Soient $n$ un entier, $\alpha$ un réel strictement positif et $G=$ $(\mathbb{Z} / n \mathbb{Z}) \times(\mathbb{Z} / n \mathbb{Z})$. Posons $b(n)=\{(x, y) \in G: x \wedge n=1$ et $\langle(x, y)\rangle \cap$ $\left.B^{*}(0, \alpha \sqrt{n})=\emptyset\right\}$. On $a$

$$
\operatorname{card}(b(n)) \geq n \phi(n)-4 \alpha^{2} \sigma(n) \phi(n)-2 \alpha d(n) \phi(n) \sqrt{n} .
$$

Démonstration. Notons $m(n)=\{(x, y) \in G, x \wedge n=1$ et $\langle(x, y)\rangle \cap$ $\left.B^{*}(0, \alpha \sqrt{n}) \neq \emptyset\right\}$. On a

$$
\operatorname{card}(b(n))=n \phi(n)-\operatorname{card}(m(n)) .
$$

Majorons le cardinal de $m(n)$. Soit $x \in \mathbb{Z} / n \mathbb{Z}$ tel que $x \wedge n=1$. Posons

$$
m(x)=\left\{y \in \mathbb{Z} / n \mathbb{Z}:\langle(x, y)\rangle \cap B^{*}(0, \alpha \sqrt{n}) \neq \emptyset\right\},
$$

on a

$$
\begin{aligned}
y \in m(x) & \Leftrightarrow \exists(a, b) \in B^{*}(0, \alpha \sqrt{n}) \exists k \in \mathbb{Z}, a=k x \text { et } b=k y \\
& \Leftrightarrow \exists(a, b) \in B^{*}(0, \alpha \sqrt{n}), b=\left(x^{-1} y\right) a,
\end{aligned}
$$

donc $m(x)$ est en bijection avec

$$
M=\left\{z \in \mathbb{Z} / n \mathbb{Z}: \exists(a, b) \in B^{*}(0, \alpha \sqrt{n}), b=z a\right\} .
$$

On a donc $\operatorname{card}(m(n))=\phi(n) \operatorname{card}(M)$.

Il nous faut majorer le cardinal de $M$. A chaque $(a, b) \in B^{*}(0, \alpha \sqrt{n})$ on peut associer l'ensemble $E(a, b)=\{z \in \mathbb{Z} / n \mathbb{Z}: b=z a\}$. On a

$$
\operatorname{card}(M) \leq \sum_{(a, b) \in B^{*}(0, \alpha \sqrt{n})} \operatorname{card}(E(a, b)) .
$$

Soit $(a, b) \in G$ tel que l'ordre de $a$ dans $\mathbb{Z} / n \mathbb{Z}$ soit $d$. Le nombre de solutions dans $\mathbb{Z} / n \mathbb{Z}$ de l'équation $b=z a$ est $n / d$ si $b \in\langle a\rangle$, et 0 si $b \notin\langle a\rangle$. Comme $\langle a\rangle=\langle n / d\rangle$, le nombre de $b \in\langle a\rangle$ dont la distance à 0 est inférieure à $\alpha \sqrt{n}$ est $2[\alpha \sqrt{n} /(n / d)]+1$. Ainsi le nombre de couples $(a, b) \in B^{*}(0, \alpha \sqrt{n})$ tels que $a$ soit d'ordre $d$ et $b \in\langle a\rangle$, est $2[\alpha \sqrt{n} /(n / d)](1+2[\alpha \sqrt{n} /(n / d)])$. 
Finalement, on obtient

$$
\begin{aligned}
\operatorname{card}(M) & \leq \sum_{d \mid n}(n / d) 2[\alpha \sqrt{n} /(n / d)](2[\alpha \sqrt{n} /(n / d)]+1) \\
& \leq \sum_{d \mid n} 2 \alpha \sqrt{n}(2 \alpha \sqrt{n} /(n / d)+1)
\end{aligned}
$$

et

$$
\operatorname{card}(m(n)) \leq \phi(n) \sum_{d \mid n}\left(4 \alpha^{2} d+2 \alpha \sqrt{n}\right)=4 \alpha^{2} \phi(n) \sigma(n)+2 \alpha d(n) \sqrt{n} .
$$

Le lemme 4.4 est un cas particulier d'un lemme de V. Sprindžuk ([Sp], p. 17).

Lemme 4.4. Soit $(X, \mathcal{T}, \mu)$ un espace mesuré de mesure finie et $\left(A_{n}\right)$ une suite d'éléments de $\mathcal{T}$. S'il existe des constantes $c$ et $c^{\prime}>0$ telles que

$$
\forall N>0 \quad \text { (1) } \sum_{n=N}^{2 N-1} \mu\left(A_{n}\right) \geq c \quad \text { et } \quad(2) \sum_{N<p \neq q<2 N} \mu\left(A_{q} \cap A_{p}\right) \leq c^{\prime}
$$

alors

$$
\mu\left(\limsup _{n \rightarrow \infty} A_{n}\right)>0 .
$$

Démonstration. Posons

$$
E_{N}=\bigcup_{n=N}^{2 N-1} A_{n} \quad \text { et } \quad L_{N}=\sum_{n=N}^{2 N-1} \mu\left(A_{n}\right) \geq c .
$$

On a d'après l'inégalité de Schwarz

$$
\begin{aligned}
L_{N} & =\int_{E_{N}} 1 \times \sum_{n=N}^{2 N-1} 1_{A_{n}}(x) d \mu(x) \\
& \leq\left(\mu\left(E_{N}\right)\right)^{1 / 2}\left(\int_{E_{N}} \sum_{p, q} 1_{A_{p}}(x) 1_{A_{q}}(x) d \mu(x)\right)^{1 / 2} .
\end{aligned}
$$

D'où

$$
\begin{aligned}
\mu\left(E_{N}\right) & \geq L_{N}^{2} /\left(L_{N}+\sum_{p \neq q} \mu\left(A_{q} \cap A_{p}\right)\right) \\
& \geq L_{N}\left(1+c^{\prime} / L_{N}\right)^{-1} \geq c\left(1+c^{\prime} / c\right)^{-1}=a>0 .
\end{aligned}
$$

On conclut en remarquant que

$$
\limsup _{n \rightarrow \infty} E_{2^{n}} \subseteq \limsup _{n \rightarrow \infty} A_{n}
$$

et que

$$
\mu\left(\limsup _{n \rightarrow \infty} E_{2^{n}}\right)=\lim _{p \rightarrow \infty} \mu\left(\bigcup_{n>p} E_{2^{n}}\right) \geq a .
$$


Lemme 4.5. Soient $N, n$ et $m$ des entiers tels que $n, m \in\{N, \ldots, 2 N\}$ et $n>m$. Soit $c>0$. Posons

$I(n, m)=\left\{p \in\{0, \ldots, n-1\}: \exists q \in\{0, \ldots, m-1\},\|p / n-q / m\| \leq c N^{-3 / 2}\right\}$.

Si l'ordre de $m$ dans $\mathbb{Z} / n \mathbb{Z}$ est $d$, où d est un diviseur de $n$, alors on a

$$
\operatorname{card}(I(n, m)) \leq n / d+8 c N^{1 / 2} .
$$

Démonstration. On a

$$
I(n, m) \subseteq\left\{p \in\{0, \ldots, n-1\}: \exists q \in\{0, \ldots, m\},|p m-q n| \leq 4 c N^{1 / 2}\right\} .
$$

Appelons $\delta$ la distance naturelle dans $\mathbb{Z} / n \mathbb{Z}$. On a donc

$$
\operatorname{card}(I(n, m)) \leq \operatorname{card}\left(\left\{p \in\{0, \ldots, n-1\}: \delta(0, p m) \leq 4 c N^{1 / 2}\right\}\right) .
$$

Comme l'ordre de $m$ est $d$, on a au plus $8 c N^{1 / 2} /(n / d)+1$ éléments de $\langle m\rangle$ dans la boule de centre 0 et rayon $4 c N^{1 / 2}$, donc

$$
\begin{aligned}
\operatorname{card}(I(n, m)) & \leq \operatorname{card}\left(\left\{p \in\{0, \ldots, n-1\}: \delta(0, p m) \leq 4 c N^{1 / 2}\right\}\right) \\
& \leq(n / d)\left(1+8 c N^{1 / 2} /(n / d)\right)=n / d+8 c N^{1 / 2} .
\end{aligned}
$$
$[\mathrm{H}, \mathrm{W}]$.

Le lemme 4.6 est une conséquence immédiate des estimations classiques

Lemme 4.6. Il existe des constantes $A$ et $a>0$ telles que pour tout entier $N$ on ait

$$
\sum_{n=N}^{2 N} \sigma(n) \leq A N^{2}, \quad \sum_{n=N}^{2 N} d(n) \leq A N^{3 / 2}, \quad \sum_{n=N}^{2 N} \phi(n) \geq a N^{2} .
$$

Lemme 4.7. L'ensemble des $\theta$ de $\mathbb{T}^{2}$ tels que $\liminf _{n \rightarrow \infty} e(n, \theta) \sqrt{n}<\infty$ est de mesure strictement positive, i.e., $\mathcal{B}$ est de mesure strictement positive.

Dém o n stration. Soient $\alpha$ et $\beta$ deux réels strictement positifs que l'on choisira plus loin. Pour $n$ entier posons $E(n)=\bigcup_{x \in b(n)} B\left(x / n, \beta n^{-3 / 2}\right)$, où on a identifié $x / n$ à un point de $\mathbb{T}^{2}(b(n)$ est défini dans le lemme 4.3). Si $x \in b(n)$ alors par définition de $b(n)$ on a $r(n-1, x / n) \geq \alpha / \sqrt{n}$, donc pour $y \in E(n)$ on a $r(n-1, y) \geq(\alpha-\beta) / \sqrt{n}>\alpha /(2 \sqrt{n})$ si $\beta<\alpha / 2$. De plus, $\|n y\| \leq \beta / \sqrt{n}<\alpha /(2 \sqrt{n})$, ainsi $n$ est une meilleure approximation de $y$ et on a d'après la proposition 2.2 ,

$$
e(n, y) \leq 3 /(n r(n-1, y)) \leq 6 /(\alpha \sqrt{n}) .
$$

Pour prouver le lemme, il suffit donc de prouver que $\lim _{\sup _{n \rightarrow \infty}} E(n)$ est un ensemble de mesure strictement positive pour un bon choix de $\alpha$ et $\beta$. Utilisons le lemme 4.4, montrons les inégalités (1) et (2) de ce lemme. On a 
d'après le lemme 4.3 ,

$$
\begin{aligned}
\sum_{n=N}^{2 N-1} \operatorname{mesure}(E(n)) & =\sum_{n=N}^{2 N-1} \beta^{2} n^{-3} \operatorname{card}(b(n)) \\
& \geq \sum_{n=N}^{2 N-1} \beta^{2} n^{-3}\left\{n \phi(n)-4 \alpha^{2} \phi(n) \sigma(n)-2 \alpha \sqrt{n} d(n) \phi(n)\right\} .
\end{aligned}
$$

Le lemme 4.6 montre que

$$
\begin{aligned}
\sum_{n=N}^{2 N-1} \beta^{2} n^{-3} & \left\{n \phi(n)-4 \alpha^{2} \phi(n) \sigma(n)-2 \alpha \sqrt{n} d(n) \phi(n)\right\} \\
& \geq \sum_{n=N}^{2 N-1} \beta^{2} N^{-3}\left\{N \phi(n)-8 \alpha^{2} N \sigma(n)-8 \alpha N^{3 / 2} d(n)\right\} \\
& \geq \beta^{2} N^{-3}\left\{a N^{3}-8 \alpha^{2} A N^{3}-8 \alpha N^{3}\right\} \geq \beta^{2}(a-16 \alpha) .
\end{aligned}
$$

La première condition du lemme 4.4 est donc satisfaite pour $\alpha$ assez petit.

Montrons que la deuxième condition du lemme 4.4 est satisfaite. Soient $n, m \in\{N, \ldots, 2 N\}$. En prenant $c=\beta$ dans le lemme 4.5 on obtient

$$
E(n) \cap E(m) \subseteq \bigcup_{x \in I(n, m) \times I(n, m)} B\left(x, \beta N^{-3 / 2}\right),
$$

et

$$
\operatorname{mesure}(E(n) \cap E(m)) \leq\left(n / d+8 \beta N^{1 / 2}\right)^{2} \beta^{2} N^{-3},
$$

où $d$ est l'ordre de $m$ dans $\mathbb{Z} / n \mathbb{Z}$. D'après le lemme 4.6 , on a

$$
\begin{aligned}
\sum_{N \leq m<n<2 N} \operatorname{mesure}(E(n) \cap E(m)) \\
\leq \sum_{N<n<2 N} \sum_{d \mid n} \phi(d)\left(n / d+8 \beta N^{1 / 2}\right)^{2} \beta^{2} N^{-3} \\
\leq \sum_{N<n<2 N} \sum_{d \mid n} d\left(n^{2} / d^{2}+64 \beta^{2} N+32 \beta N^{3 / 2} / d\right)^{2} \beta^{2} N^{-3} \\
\leq 64 \beta^{2} N^{-2} \sum_{N<n<2 N} \sum_{d \mid n}\left(n / d+\beta^{2} d+\beta N^{1 / 2}\right) \leq C,
\end{aligned}
$$

où $C$ ne dépend pas de $N$.

Et a pe 3. Soit $T$ la transformation de $\mathbb{T}^{2}$ qui envoie $x \in \mathbb{T}^{2}$ sur $2 x \in \mathbb{T}^{2}$. Alors $T$ est mélangeante et conserve la mesure de Lebesgue. Comme $B$ est de mesure strictement positive, pour montrer que $B$ est de mesure 1 , il suffit de montrer que $T(\mathcal{B}) \subseteq \mathcal{B}$. Montrons que pour $\theta \in \mathbb{T}^{2}$ on a $e(n, T(\theta)) \leq 2 e(n, \theta)$. 
Pour $y \in \mathbb{T}^{2}$ il existe $z \in \mathbb{T}^{2}$ tel que $y=2 z$. Si $k \in\{0, \ldots, n\}$ est tel que $d(z,\{0, \theta, \ldots, n \theta\})=d(z, k \theta)=\|z-k \theta\|$, on a

$$
\|y-k(2 \theta)\|=\|2(z-\theta)\| \leq 2\|z-\theta\| \leq 2 e(n, \theta),
$$

donc $e(n, 2 \theta) \leq 2 e(n, \theta)$. Donc pour tout $\theta \in \mathbb{T}^{2}$ on a

$$
\liminf _{n \rightarrow \infty} e(n, 2 \theta) \sqrt{n} \leq 2 \liminf _{n \rightarrow \infty} e(n, \theta) \sqrt{n} \quad \text { et } \quad T(\mathcal{B}) \subseteq \mathcal{B} .
$$

R e m a r qu e. Il resterait à déterminer la dimension de Hausdorff de l'ensemble exceptionnel $\mathbb{T}^{2} \backslash \mathcal{A}$.

\section{Références}

[H,W] G. H. Hardy and E. M. Wright, An Introduction to the Theory of Numbers, 4th ed., Oxford University Press, 1971.

[L] J. C. Lagarias, Best simultaneous Diophantine approximations. I. Growth rates of best approximation denominators, Trans. Amer. Math. Soc. 272 (1982), 545554 .

[So] V. T. Sós, On the distribution mod 1 of the sequence no, Ann. Univ. Sci. Budapest. Eötvös Sect. Math. 1 (1958), 127-134.

[Sp] V. Sprindžuk, Metric Theory of Diophantine Approximations, V. H. Winston \& Sons, Washington, D.C., 1979.

UNIVERSITÉ DE HAUTE ALSACE

4 RUE DES FRÈRES LUMIÈRE

68093 MULHOUSE, FRANCE 